\title{
An Engineering Approach for Elastic-Plastic J-Integral of Surface Cracks in Plates Using the Nonlinear Line-Spring Model
}

\author{
Jing $\mathrm{WU}^{1, \mathrm{a}}$, Jie-Hong YUAN ${ }^{2, \mathrm{~b} *}$ and Yuan $\mathrm{LI}^{3, \mathrm{c}}$ \\ $1,2,3$ College of Basic Education, National University of Defense Technology, Changsha, China \\ bwujingprc@foxmail.com \\ *Corresponding author
}

Keywords: J-integral, Surface crack, Nonlinear line-spring model, Work hardening material.

\begin{abstract}
In this paper, a nonlinear line-spring model with new plastic constitutive relations for the line-spring is presented based on the fully plastic solutions of the single-edge cracked strip subjected to pure tension and pure bending. Numerical solutions of $J$-integral which characterizes the amplitude of the HRR singularity field, are then calculated for a number of surface cracked plates. The numerical analysis shows that the agreement between the finite-element method and the line-spring model is remarkable. Besides, studied are the influences of the crack size and plastic work hardening exponent on the $J$ values.
\end{abstract}

\section{Introduction}

Surface cracks are common flaws encountered in structural materials, particularly in pressure vessels and pipelines. Owing to the surface crack's three-dimensional nature, there is no complete elastic-plastic analytic solution available for it. However, many of these problems can be relatively precisely estimated by a simplified method named the nonlinear line-spring model (LSM).

Recent progress in elastic-plastic fracture mechanics has made possible engineering procedures like the LSM for assessing the integrity of structural components from a viewpoint of fracture. In 1972, Rice and Levy [1] put forward a linear line-spring to calculate the stress intensity factors $\left(K_{I}\right)$ in plate or shell structures containing part-through surface cracks. In the early 1980s, Parks [2], and independently, $\mathrm{Lu}$ [3] et al. extended this useful model into the analysis of elastic and plastic surface cracks by introducing a nonlinear line-spring model. The past few decades have witnessed continual attentions of researchers to the LSM and this model has been improved from different aspects so as to be more in line with three-dimensional nature of surface cracked materials [4-7].

The plastic constitutive relations for the line-spring developed by Lu et al. depend on fully plastic solutions of single-edge cracked plate (SECP) under tension tabulated in handbook format [8], the numerical procedure of which is specifically designed for two-dimensional problems and cannot be generalized readily to three dimensions [9]. In this paper, it is presented a nonlinear line-spring model with new plastic constitutive relations for the line-spring based on Shih's fully plastic solutions of SECP subjected to pure tension and pure bending using reduced integration penalty methods [9]. Using this model, $J$-integral values are calculated in some surface crack configurations. The numerical analysis by the LSM is compared with the finite element method solution. Also analyzed are the effects of the crack size and plastic work hardening exponent on the $J$-integral values.

\section{Nonlinear Constitutive Relations for the Line-spring of Work Hardening Materials}

The semi-elliptical surface crack of depth $a$ and length $2 c$ as shown in Fig. 1(a) is replaced with a through crack of length $2 c$ where distributed line-springs connecting two surfaces of the crack is equivalent to the constraints from the non-cracked ligament, as shown in Fig. 1(b). The nonlinear 
constitutive relations of line-spring are simulated by a plane-strain SECP of thickness $t$ and crack depth $a$, as Fig. 1(c) shows.

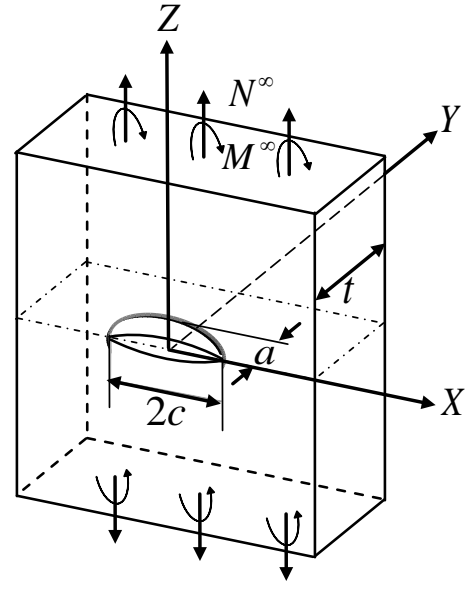

(a)

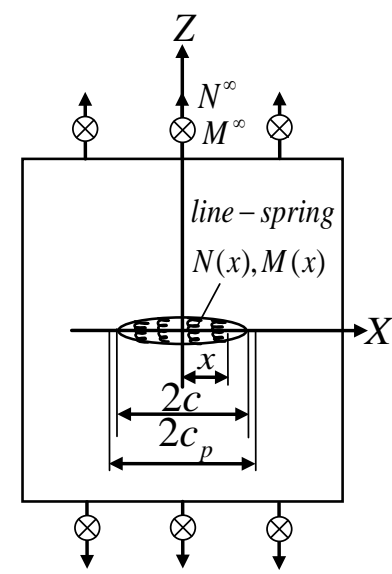

(b)

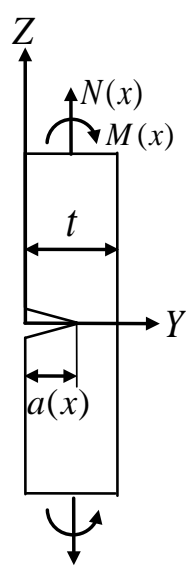

(c)

Fig. 1 Semi-elliptical surface crack replaced by a through crack with distributed line-springs.

For the SECP subjected to combined load of membrane force $N(x)$ and bending moment $M(x)$, the $J$-integral of crack tip and the additional stretch $\delta$ or rotation $\theta$ due to the existence of crack can be expressed by the complementary strain energy $\Omega$

$J=\partial \Omega / \partial a$,

$\delta=\partial \Omega / \partial N, \quad \theta=\partial \Omega / \partial M$

The line-spring model assumes that the uniaxial tension properties of materials should obey Ramberg-Osgood type stress-strain relationship, which can be expressed as

$\varepsilon / \varepsilon_{s}=\sigma / \sigma_{s}+\alpha\left(\sigma / \sigma_{s}\right)^{n}$.

where $\alpha$ and $n$ are material constants, $\sigma_{s}$ is the yield stress, $\varepsilon_{s}=\sigma_{s} / E, E$ is Young's modulus of elasticity. The complementary strain energy $\Omega$ and the additional stretch $\delta$ or rotation $\theta$ can be decomposed into linear elastic and plastic parts

$\Omega=\Omega^{e}+\Omega^{p}$,

$\delta=\delta^{e}+\delta^{p}, \theta=\theta^{e}+\theta^{p}$

Rice [1] obtained the elastic generalized displacement formula in the calculation of stress intensity factors for part-through surface cracks, which may be written as

$$
\left\{\bar{q}^{e}\right\}=\frac{2 \sigma_{s}\left(1-v^{2}\right)}{E}\left[\begin{array}{ll}
\alpha_{M M} & \alpha_{M B} \\
\alpha_{B M} & \alpha_{B B}
\end{array}\right]\{\bar{\sigma}\} .
$$

where $\left\{\bar{q}^{e}\right\}^{T}=\left[\begin{array}{ll}\bar{\delta}^{e} & \bar{\theta}^{e}\end{array}\right]=\left[\begin{array}{ll}\delta^{e} / t & \theta^{e} / 6\end{array}\right], \quad\{\bar{\sigma}\}^{T}=\left[\begin{array}{ll}\bar{\sigma}_{M} & \bar{\sigma}_{B}\end{array}\right]=\left[\begin{array}{ll}N(x) / t \sigma_{s} & 6 M(x) / t^{2} \sigma_{s}\end{array}\right]$, $\alpha_{\lambda \mu}=\int_{0}^{\xi_{e}} g_{\lambda}(\xi) g_{\mu}(\xi) d \xi \quad(\lambda, \mu=M, B), \xi=a / t, \xi_{e}=a_{e} / t, g_{M}(\xi)$ and $g_{B}(\xi)$ may be taken from Ref. [10], $a_{e}$ is the Irwin's equivalent crack size presented as equal to the original crack depth plus a plastic zone correction and we employ the effective length derived by Shih [11] et al. 
The plastic constitutive relations are investigated by the plastic complementary strain energy $\Omega^{p}$. Similar to the method performed by Miyoshi [4], the complementary energy $\Omega^{p}$ for this problem is assumed as

$$
\begin{aligned}
& \Omega^{p}=\frac{\alpha \varepsilon_{s} \sigma_{s}}{n+1} t^{2} f^{(n+1) / 2}, \\
& f=\frac{A N(x)^{2}+B N(x) M(x) / t+C(M(x) / t)^{2}}{\sigma_{s}{ }^{2}(t-a)^{2}}=\frac{A \bar{\sigma}_{M}{ }^{2}+B \bar{\sigma}_{M} \bar{\sigma}_{B} / 3+C \bar{\sigma}_{B}{ }^{2} / 36}{(1-\xi)^{2}} .
\end{aligned}
$$

The $A, B$ and $C$ are undetermined coefficients relevant to crack sizes and material properties. And we have

$$
\begin{aligned}
& \delta^{p}=\frac{\partial \Omega^{p}}{\partial N}=\frac{\alpha}{(1-\xi)^{2} E}(A N(x)+B M(x) / t) f^{(n-1) / 2}, \\
& \theta^{p}=\frac{\partial \Omega^{p}}{\partial M}=\frac{\alpha}{(1-\xi)^{2} t E}(B N(x)+C M(x) / t) f^{(n-1) / 2} .
\end{aligned}
$$

The full plastic numerical solutions for a wide variety of surface crack configurations are put forward by Shih [9] et al. using an incompressible finite-element penalty method applicable to plane-strain problem. The following equations are given

$$
\left.\begin{array}{l}
\delta^{p}=\alpha \varepsilon_{s} a h_{3 N}(\xi, n)\left(N / N_{0}\right)^{n} \\
\theta^{p}=\alpha \varepsilon_{s} h_{5 N}(\xi, n)\left(N / N_{0}\right)^{n}
\end{array}\right\} \quad \begin{array}{r}
\text { pure tension, } \\
\theta^{p}=\alpha \varepsilon_{s} h_{3 M}(\xi, n)\left(M / M_{0}\right)^{n}
\end{array} \quad \text { pure bending. }
$$

where $N_{0}=1.455 \eta t \sigma_{s}$ with $\eta=\sqrt{(1-\xi)^{2}+\xi^{2}}-\xi, M_{0}=0.364(1-\xi)^{2} t^{2} \sigma_{s}$.

In Eq. 8, order $N=0$ and $M=0$, then compare them with Eq. 9, gives

$$
\begin{aligned}
& A=\left[\xi h_{3 N}(\xi, n)(1-\xi)^{n+1} /(1.455 \eta)^{n}\right]^{2 /(n+1)}, \\
& B=A h_{5 N}(\xi, n) / \xi h_{3 N}(\xi, n), \\
& C=\left[h_{3 M}(\xi, n) / 0.364^{n}(1-\xi)^{n-1}\right]^{2 /(n+1)} .
\end{aligned}
$$

where $h_{3 N}(\xi, n), h_{5 N}(\xi, n), h_{3 M}(\xi, n)$ are tabulated in Ref. [9].

Adopting the normalization, the plastic generalized displacement formula is

$$
\left\{\bar{q}^{p}\right\}=\frac{\alpha \sigma_{s}}{(1-\xi)^{2} E} f^{(n-1) / 2}\left[\begin{array}{cc}
A & B / 6 \\
B / 6 & C / 36
\end{array}\right]\{\bar{\sigma}\} .
$$

where $\left\{\bar{q}^{p}\right\}^{T}=\left[\begin{array}{ll}\bar{\delta}^{p} & \bar{\theta}^{p}\end{array}\right]=\left[\begin{array}{ll}\delta^{p} / t & \theta^{p} / 6\end{array}\right]$.

Thus, the equation 6 and 11 constitute the nonlinear elastic-plastic constitutive relation, gives

$$
\{\bar{q}\}=\left\{\bar{q}^{e}\right\}+\left\{\bar{q}^{p}\right\}=\frac{2 \sigma_{s}\left(1-v^{2}\right)}{E}\left[\begin{array}{ll}
\alpha_{M M} & \alpha_{M B} \\
\alpha_{B M} & \alpha_{B B}
\end{array}\right]\{\bar{\sigma}\}+\frac{\alpha \sigma_{s}}{(1-\xi)^{2} E} f^{(n-1) / 2}\left[\begin{array}{cc}
A & B / 6 \\
B / 6 & C / 36
\end{array}\right]\{\bar{\sigma}\} .
$$




\section{Nonlinear Equations of the Nonlinear Line-spring Model}

The D-M model solutions for infinitely wide thin plate with a through crack, as shown in Fig. 1(b), are obtained by Lu [3] et al. Through the use of revised limited-width plate, with the actual sizes, the equations are given by

$$
\{\bar{q}\}=\frac{4 \sigma_{s} c f_{\text {limited-width }}}{E t}\left[\begin{array}{ll}
1 & 0 \\
0 & \gamma
\end{array}\right]\left[I(\bar{x})\left\{\bar{\sigma}^{\infty}\right\}-\int_{0}^{1} H(\bar{x}, \bar{t})\{\bar{\sigma}\} d \bar{t}\right],
$$

where $I(\bar{x})=\sqrt{{\overline{c_{p}}}^{2}-\bar{x}^{2}}-\pi G\left(\bar{x}, \bar{c}_{p}\right) / 2, G\left(\bar{x}, \bar{c}_{p}\right)=\int_{1}^{c_{p}} F(\bar{x}, \bar{t}) d \bar{t} / S \quad, \quad S=\pi / 2-\arcsin \left(1 / \bar{c}_{p}\right)$,

$$
\begin{aligned}
& \bar{x}=x / c \quad, \quad \bar{t}=t / c, \quad \bar{c}_{p}=c_{p} / c, \quad H(\bar{x}, \bar{t})=F(\bar{x}, \bar{t})-G\left(\bar{x}, \bar{c}_{p}\right) / \sqrt{\bar{c}_{p}{ }^{2}-\bar{t}^{2}}, \\
& \left\{\bar{\sigma}^{\infty}\right\}^{T}=\left[N^{\infty} / t \sigma_{s} \quad 6 M^{\infty} / t^{2} \sigma_{s}\right] \\
& F(\bar{x}, \bar{t})=\ln \left|\left(\sqrt{\bar{c}_{p}^{2}-\bar{x}^{2}}+\sqrt{\bar{c}_{p}{ }^{2}-\bar{t}^{2}}\right) /\left(\sqrt{\bar{c}_{p}^{2}-\bar{x}^{2}}-\sqrt{\bar{c}_{p}{ }^{2}-\bar{t}^{2}}\right)\right| / \pi, \gamma=(1+v) /(9+3 v), \quad v \quad \text { is } \\
& \text { Poisson's ratio, } \quad f_{\text {limited-width }}=h_{2}(\pi a c / 2 b t, n) / 4(1-\pi a c / 2 b t) \quad \text { is the limited-width }
\end{aligned}
$$
coefficient, $h_{2}(\pi a c / 2 b t, n)$ is presented in Ref. [8], and

$$
\left(\pi \bar{\sigma}_{M}^{\infty} / 2-\int_{0}^{1} \bar{\sigma}_{M} / \sqrt{{\overline{c_{p}}}^{2}-\bar{x}^{2}} d \bar{x}\right)^{2} / S^{2}+2\left|\pi \bar{\sigma}_{B}^{\infty} / 2-\int_{0}^{1} \bar{\sigma}_{B} / \sqrt{{\overline{c_{p}}}^{2}-\bar{x}^{2}} d \bar{x}\right| / 3 S=1 .
$$

The equation 12, 13 and 14 constitute the basic incremental expression for the elastic-plastic fracture analysis of a cracked plate with finite-dimensions.

\section{Numerical Results and Discussion}

The elastic-plastic $J$-integral for a thin plate with semi-elliptical surface crack under combined tension and bending is obtained as

$J=J^{e}\left(a_{e}\right)+J^{p}$,

with $J^{e}\left(a_{e}\right)=\left(1-v^{2}\right) K_{I}^{2} / E$ and $J^{p}=\partial \Omega^{p} / \partial a$.

Adopting the normalized value of the total $J$-integral, we have

$$
\bar{J}=\frac{J E}{t \sigma_{s}^{2}}=\left(1-v^{2}\right)\left[\bar{\sigma}_{M} g_{M}\left(\xi_{e}\right)+\bar{\sigma}_{B} g_{B}\left(\xi_{e}\right)\right]^{2}+\frac{\alpha}{2}\left(A^{\prime} \bar{\sigma}_{M}{ }^{2}+\frac{B^{\prime}}{3} \bar{\sigma}_{M} \bar{\sigma}_{B}+\frac{C^{\prime}}{36} \bar{\sigma}_{B}^{2}\right) f^{(n-1) / 2} \text {. }
$$

where $A^{\prime}=\left[A /(1-\xi)^{2}\right]_{\xi}^{\prime}, B^{\prime}=\left[B /(1-\xi)^{2}\right]_{\xi}^{\prime}, C^{\prime}=\left[C /(1-\xi)^{2}\right]_{\xi}^{\prime}$.

In order to quantitatively demonstrate the accuracy of the nonlinear LSM proposed, an example of a plate with thickness $t$, height $h / t=32$ and width $b / t=16$ in the Ref. [6] are selected. The plate contains a surface crack with maximum depth of $a / t=0.6$ and opening length of $a / c=0.24$. The ends of the plate are subjected to remote uniform uniaxial $N^{\infty}$ and the mechanical properties of materials are set to the following values: $\alpha=1.0, \sigma_{s}=452[\mathrm{MPa}, E=200$ [GPa], $v=0.3$, and $n=3$. The finite-element calculations by Shawki [6] are carried out with $h / t=32$; however, previous numerical results have proved that it is sufficient to eliminate any dependence of interest on the ratio. The values of the $J$-integral at the center line (the deepest point of the crack tip) obtained from the finite element method and the LSM are plotted in Fig. 2, against normalized tensile load $N / N_{0}$, where $N_{0}=\sigma_{s}(b t-\pi a c / 2)$. From Fig. 2, it is seen that the values of the deepest point's $J$-integral obtained in this work agree well with those calculated by the finite-element method [6]. 


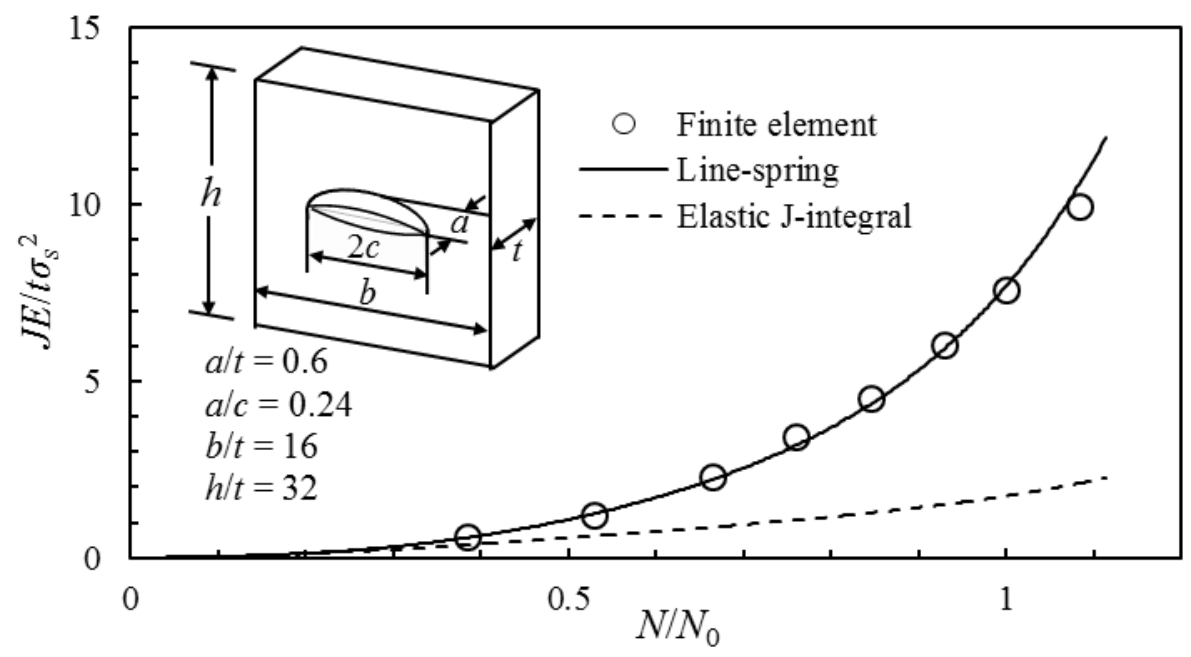

Fig. 2 Comparisons of normalized $J$ solutions from LSM and finite-element vs. normalized tensile.

The values of four group fractions calculated for $J$-integral in some surface crack configurations are shown in Fig. 3. As can be seen, the load-normalized $J$ remains almost constant at low stress levels and the normalized $J$ rises rapidly with the increasing remote load. Also shown in Fig. 2 are the elastic $J$-integral $\left(\bar{J}^{e}(a)\right)$ values contributing significantly to the total $J$-integral values at low stress levels, and under high stress levels it seems reasonable to conclude that the $J$-integral's behavior is dominantly plastic. Fig. 3 also shows that at low values of remote load, the high hardening normalized $J$ values are larger than those of the low hardening and surface crack with shorter opening length representing higher $J$ values.

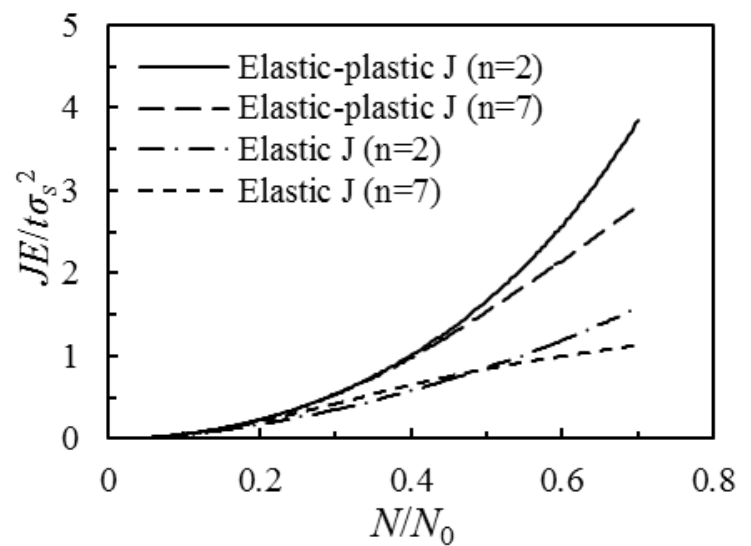

(a) $a / c=0.2, a / t=0.625, c / b=0$

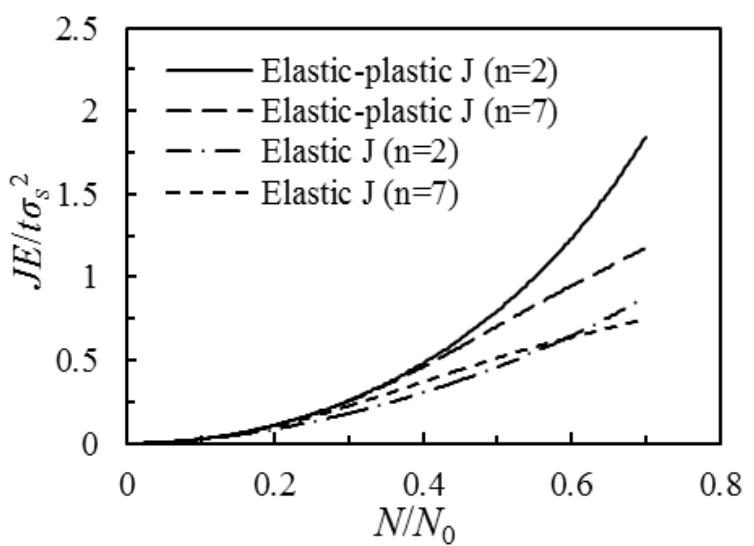

(b) $a / c=0.4, a / t=0.625, c / b=0$

Fig. 3 Normalized $J$ solutions vs. normalized tensile load in some surface crack configurations.

\section{Summary}

In this paper, a new nonlinear line-spring model is established for the elastic-plastic numerical analysis of surface cracks with particular reference to the work hardening materials. The plastic constitutive relations for the line-spring are reconstituted by the plastic complementary strain energy for the single-edge cracked strip subjected to combined tension and bending based on Shih's fully plastic solutions of the SECP under pure tension and pure bending. And it appears that the new line-spring model gives reasonably accurate estimates of $J$-integral for surface cracks. However, because only seven data points are tabulated in Ref. [9], the fitting errors of the $h$ functions will directly influence the computational accuracy. Furthermore, the results of four group fractions are 
calculated for $J$-integral in some surface crack configurations. In the further, the LSM method can readily assess the $3^{\text {rd }}$ kind choice-curve with the development of its accuracy.

\section{Acknowledgment}

This research is supported by the National Natural Science Foundation of China (No. 11402297), the Science Fund of State Key Laboratory of Advanced Design and Manufacturing for Vehicle Body (No. 51315005), the Science Fund of State Key Laboratory of Traction Power (No.TPL1409).

\section{References}

[1] J. R. Rice and N. Levy. The part-through surface crack in an elastic plate. Journal of Applied Mechanics 39.1(1971):185-194.

[2] D. M. Parks. The inelastic line-spring: estimates of elastic-plastic fracture mechanics parameters for surface-cracked plates and shells. Journal of Pressure Vessel Technology 103.3(1981):246-254.

[3] Y. C. Lu and J. Ning. The nonlinear line-spring model for surface crack analysis with particular reference to work hardening materials. Proc. of The ICF International Symposium On Fracture Mechanics, Beijing, 1983.

[4] T. Miyoshi, M. Shiratori and Y. Yoshida. Analysis of J-integral and crack growth for surface cracks by line spring method. Journal of Pressure Vessel Technology 108.3(1986):305-311.

[5] Y. C. Lu and C. Guo. A new nonlinear line-spring model for elastic-plastic analysis of surface cracks. Proc. of The 7th International Conference On Fracture (ICF7), (1989):525-531.

[6] T. G. Shawki, T. Nakamura and D. M. Parks. Line-spring analysis of surface flawed plates and shells using deformation theory. International Journal of Fracture 41.1(1989):23-38.

[7] Z. Z. Huang and Q. Gao. Evaluation of the J-integral for surface cracks using the line-spring model. Engineering Fracture Mechanics (1994):251-263.

[8] V. Kumar, M. D. German and C. F. Shih. Engineering approach for elastic-plastic fracture analysis. EPRI Report NP-1931, Electric Power Research Institute 94(1981).

[9] C. F. Shih and A. Needleman. Fully plastic crack problems, Part 1: solutions by a penalty method. Journal of Applied Mechanics 51.1(1984):48-56.

[10] H. Tada, P. C. Paris and G. R. Irwin. The stress analysis of cracks handbook, 1973. Stress Analysis of Cracks Handbook 91.181(1973):614.

[11] C. F. Shih, W. R. Andrews, H. G. Delorenzi et al. Methodology for plastic fracture.Final report. General Electric Final Report to Electric Power Research Institute, Publication No.EPRI-1735 (1981). 